\title{
Diagnosis of atrial fibrillation in heart failure patients with implantable cardioverter defibrillator or cardiac resynchronisation therapy
}

\author{
Barbara Dominik ${ }^{1}$, Przemyslaw Mitkowski², Wojciech Zorawski ${ }^{3}$, Ilona Kowalik ${ }^{4}$ Adam Ciesielski ${ }^{3}$
}

\author{
${ }^{1}$ Department of Internal Medicine, University Hospital, Zielona Gora, Poland \\ ${ }^{2}$ Department of Cardiology, University Hospital, Poznan, Poland \\ ${ }^{3}$ Department of Cardiology, Multidisciplinary Hospital, Nowa Sol, Poland \\ ${ }^{4}$ Institute of Cardiology, Warsaw, Poland
}

Submitted: 8 October 2019; Accepted: 1 December 2019 Online publication: 25 March 2021

Arch Med Sci 2021; 17 (6): 1583-1589

DOI: https://doi.org/10.5114/aoms/114977

Copyright $\odot 2021$ Termedia \& Banach

\author{
Corresponding author: \\ Barbara Dominik MD \\ Department of Internal \\ Medicine \\ University of Zielona Gora \\ Zielona Gora, Poland \\ E-mail: barbaradominik48@ \\ gmail.com
}

\begin{abstract}
Introduction: Implantable cardioverter defibrillators register various types of arrhythmias. Thus they can be exploited to better identify patients with atrial fibrillation episodes and increase the proportion of patients who may benefit from implementation of pharmacological prophylaxis of thromboembolic events, most of which are asymptomatic. The aim of the study was to assess of the frequency, symptoms and predisposing factors for the occurrence of atrial fibrillation episodes in patients with an implanted implantable cardioverter defibrillator (ICD) and cardiac resynchronisation therapy with defibrillator (CRT-D) based on the analysis of intracardiac electrocardiograms (EGM/IEGM) records.

Material and methods: The study included 174 consecutive outpatients with heart failure, sinus rhythm and an implanted cardioverter defibrillator and cardiac resynchronisation therapy with defibrillator. Follow-up visits with analysis of IEGM records occurred every 3 months. During a mean follow-up of 20 months, 901 visits were carried out. One hundred forty-seven patients had at least 1 year of follow-up.

Results: Atrial fibrillation episodes in the study group occurred in 54 (31.0\%) patients and $71.4 \%$ were asymptomatic. Predisposing factors were: history of paroxysmal atrial fibrillation ( $37.0 \%$ vs. $13.3 \%, p<0.001)$, atrioventricular conduction abnormalities ( $42.6 \%$ vs. $20.0 \%, p=0.002)$, intraventricular conduction abnormalities ( $59.3 \%$ vs. $40.8 \%, p=0.02)$ and more severe mitral regurgitation $(7.4 \%$ vs. $0.8 \%, p=0.04)$. Chronic renal disease was a risk factor for death in the study group. No stroke occurred during the study. Conclusions: Episodes of paroxysmal atrial fibrillation in patients with systolic heart failure and implanted cardioverter-defibrillator systems are quite common. The majority of the episodes recorded in the study were asymptomatic.
\end{abstract}

Key words: atrial fibrillation episode, intracardiac electrocardiogram.

\section{Introduction}

Implantable cardioverter-defibrillator and resynchronisation therapy devices have the ability to register various types of arrhythmias. Many models, apart from detecting life-threatening ventricular arrhythmias, also detect paroxysmal atrial arrhythmias, including atrial fibrillation. 
This allows for better identification of patients with atrial fibrillation (AF) episodes and thus an increase in the proportion of patients who will be able to implement pharmacological prophylaxis of thromboembolic events, especially stroke. Analysis of the intracardiac electrocardiogram should be part of every ambulatory follow-up patient visit, because many of these AF episodes are asymptomatic.

\section{Material and methods}

A series of 174 patients after cardioverter-defibrillator implantation (including resynchronisation systems) were included in the study and were undergoing follow-up visits at the outpatient clinic. Recruitment was continued until AF was diagnosed during follow-up in the $50^{\text {th }}$ subject based on memory analysis of devices and/or clinical data. The exclusion criterion was AF at the time of the screening visit. The study protocol was approved by the local ethics committee at the University of Medical Sciences (No. 601/14).

Analyses of clinical parameters and memory contents of devices were performed during ambulatory follow-up visits, which took place every $3 \pm 1$ months.

Each AF episode stored in the device's memory lasting at least $30 \mathrm{~s}$ was considered an episode of atrial fibrillation. A symptomatic episode was considered when arrhythmia led to ICD shock, heart deterioration, collapse or fainting, palpitations, weakness, chest pain or shortness of breath accompanied by a feeling of irregular heartbeat.

At the baseline visit basic demographic and clinical data were collected: age, patient's sex, date of implantation and device type, etiology and NYHA heart functional class, history of myocardial infarction, hypertension, diabetes, chronic obstructive pulmonary disease (COPD), previous stroke/transient ischemic attack (TIA), renal failure, previous AF history and indications for device implantation. After physical examination, resting ECG was recorded, the device was checked, the basic echocardiographic parameters were evaluated and the information about current pharmacological treatment was obtained.

During subsequent follow-up visits the current cardiac rhythm and interrogated device memory were analyzed.

The devices were checked appropriately using the programmers of the following companies: Biotronik, Medtronic, Boston Scientific and Abbott Laboratories. Each time, the evaluation of reports on device operation and intracardiac electrocardiogram recording was carried out by a physician trained in the assessment of cardiac implantable electronic devices. In patients with single-chamber cardioverters, AF was diagnosed, upon the analysis of device memory and recorded IEGM of rapid ventricular rhythms, detected in the programmed zones ventricular tachycardia (VT), ventricular fibrillation (VF) and "Monitor". The duration of the AF episode in these patients was the time when tachyarrhythmia was above the threshold for detection of ventricular arrhythmia, and was recorded as IEGM and qualified by the experienced physician as atrial fibrillation. Ventricular high-rate (VHR) episodes (ventricular rate $>162$ bpm) were also used to identify AF in single chamber devices.

Echocardiography was performed using ultrasound Philips EPIQ 7C device. The images were obtained from parasternal projection (long and short axis) and apical projection (two- and four-chamber). The dimensions of the left atrium (LA) and left ventricle (LV), LV ejection fraction and mitral regurgitation were assessed in accordance with the standard protocol; end-systolic volume (LVESV) and end-diastolic volume (LVEDV) of the left ventricle were calculated based on the modified Simpson method (Simpson's method biplane) using a two- and four-chamber apical projection [1]. The degree of mitral regurgitation was based on visualization using color Doppler and according to the mitral reversal range, classified according to the three-point scale: first degree - mild, second degree - moderate, third degree - severe [2].

\section{Statistical analysis}

Quantitative variables subject to normal distribution are presented as the mean and standard deviation. To assess the significance of differences between mean values of these variables, the Student's $t$-test was used. Quantitative variables with irregular distributions were characterized by means of median and interquartile range. The non-parametric Mann-Whitney test was used to verify the homogeneity of the distribution of these variables. The nominal variables are presented in the form of absolute numbers and relative frequency (\%) of the distinguished feature variants. Significance of differences between the variable fractions of different groups was estimated by Pearson's chi-squared test, with the correction for Yates or the Fisher exact test. Changes in the probability of survival without the onset of AF as a function of the time of the analyzed group of patients were determined by the Kaplan-Meier method. Uniformity of curves obtained for different subpopulations was compared by the log-rank test, with the post-hoc Tukey's test in the case of multiple comparisons (pairs of curves). In order to indicate independent predictors of AF a multivariate Cox proportional hazard analysis was used. The results are presented in the form of risk co- 
efficients with 95\% confidence intervals. Logistic regression were used to indicate independent predictors of death. Variable selection was made using the stepwise reverse method. The results are presented in the form of odds ratios together with $95 \%$ confidence intervals. Bilateral neutral hypotheses were verified at the statistical significance level $p<0.05$.

\section{Results}

The study included 174 consecutive patients (mean age: $64.3 \pm 10.0$ years), with heart failure in NYHA functional class I-IV implanted with ICDVR (VR - single-chamber ICD), ICD-DR (DR - dual-chamber ICD) and (RT-D. Follow-up visits were performed between 1/9/2014 and 30/04/2016. The study group made a total of 901 visits. The largest group of patients $(95 ; 54.6 \%)$ were implanted with a single chamber cardioverter defibrillator ICD-VR, 50 (28.7\%) patients underwent resynchronization therapy with a defibrillator (CRT-D), and 29 (16.7\%) patients were implanted with a dual chamber ICD.

In the current study $31 \%$ of patients experienced AF episodes and $71.4 \%$ of them were asymptomatic.

The demographic and clinical characteristics of the study group and drug therapy are shown in Table I.

Significantly increased risk of AF was demonstrated by patients with atrioventricular conduction disorders $(p=0.002)$, intraventricular conduction disturbances ( $p=0.02)$, a history of AF $(p<0.001)$ and more severe mitral regurgitation $(p=0.04)$.

Table I. Demographic and clinical characteristics of all patients and drug therapy

\begin{tabular}{|c|c|c|c|c|}
\hline Parameter & $\begin{array}{c}\text { All } \\
N=174\end{array}$ & $\begin{array}{c}\text { AF'‘ }^{\prime}- \\
n=120(69.0 \%)\end{array}$ & $\begin{array}{c}\mathrm{AF}^{\prime}+{ }^{\prime} \\
n=54(31.0 \%)\end{array}$ & $P$-value \\
\hline Age & $64.3 \pm 10.0$ & $63.8 \pm 10.0$ & $65.5 \pm 10.0$ & 0.28 \\
\hline Male sex & $127(73.0)$ & $86(71.7)$ & $41(75.9)$ & 0.56 \\
\hline Chronic ischemic heart disease & $123(70.7)$ & $83(69.2)$ & $40(74.1)$ & 0.51 \\
\hline Hypertension & $104(59.8)$ & $72(60.0)$ & $32(59.3)$ & 0.93 \\
\hline Diabetes & $64(36.8)$ & $44(38.7)$ & $20(37.0)$ & 0.96 \\
\hline Chronic kidney disease & $63(36.2)$ & $42(35.0)$ & $21(38.9)$ & 0.62 \\
\hline COPD & $21(12.1)$ & $15(12.5)$ & $6(11.1)$ & 0.79 \\
\hline History of stroke & $18(10.3)$ & $14(11,7)$ & $4(7.4)$ & 0.38 \\
\hline Ischemic cardiomyopathy & $118(67.8)$ & $80(66.7)$ & $38(70.4)$ & 0.63 \\
\hline Ejection fraction (\%) & $33.6 \pm 10.8$ & $33.4 \pm 11.0$ & $34.1 \pm 10.3$ & 0.70 \\
\hline LVEDD $[\mathrm{cm}]$ & $6.0 \pm 0.98$ & $6.0 \pm 1.05$ & $6.1 \pm 0.84$ & 0.85 \\
\hline Dimension of the left atrium $[\mathrm{cm}]$ & $4.6 \pm 0.67$ & $4.6 \pm 0.66$ & $4.7 \pm 0.70$ & 0.35 \\
\hline Previous atrial fibrillation & $36(20.7)$ & $16(13.3)$ & $20(37.0)$ & $<0.001$ \\
\hline Primary prevention & $137(78.7)$ & $98(81.7)$ & $39(72.2)$ & 0.17 \\
\hline Atrioventricular blocks & $47(27.0)$ & $24(20.2)$ & $23(42.6)$ & 0.002 \\
\hline Intraventricular conduction blocks & $81(46.5)$ & $49(40.8)$ & $32(59.3)$ & 0.02 \\
\hline Mitral regurgitation mild & $28(16.1)$ & $22(18.3)$ & $6(11.1)$ & \multirow[t]{3}{*}{0.04} \\
\hline Mitral regurgitation moderate & $141(81.0)$ & $97(80.8)$ & $44(81.5)$ & \\
\hline Mitral regurgitation severe & $5(2.9)$ & $1(0.8)$ & $4(7.4)$ & \\
\hline$\beta$-Blocker & $170(97.7)$ & $116(96.7)$ & $54(100)$ & 0.31 \\
\hline ACEI & $129(74.1)$ & $91(75.8)$ & $38(70.4)$ & 0.45 \\
\hline ARB & $32(18.4)$ & $18(15.0)$ & $14(25.9)$ & 0.09 \\
\hline Diuretics & $139(79.9)$ & $96(80.0)$ & $43(79.6)$ & 0.96 \\
\hline Statins & $144(82.8)$ & $100(83.3)$ & $44(81.5)$ & 0.77 \\
\hline Anticoagulant drugs & $47(27.0)$ & $20(16.7 \%)$ & $27(50)$ & $<0.001$ \\
\hline Digoxin & $25(14.4)$ & $20(16.7)$ & $5(9.3 \%)$ & 0.18 \\
\hline Ca-blocker & $20(11.5)$ & $15(12.5)$ & $5(9.3)$ & 0.53 \\
\hline Amiodarone & $33(19.0)$ & $23(19.2)$ & $10(18.5)$ & 0.92 \\
\hline Other antiarrhythmic drugs & $1(0.6)$ & $1(0.8)$ & $0(0)$ & 1.00 \\
\hline
\end{tabular}

Results presented as mean \pm SD or n (\%). COPD - chronic obstructive pulmonary disease, LVEDD - left ventricular end diastolic dimension, $A C E I$ - angiotensin-converting enzyme inhibitors, $A R B$ - angiotensin receptor blockers. 
Table II. Multivariate analysis of the risk of atrial fibrillation events

\begin{tabular}{|lccc|}
\hline Parameter & HR & $95 \% \mathrm{Cl}$ & $P$-value \\
\hline History of AF & 2.60 & $1.49-4.52$ & $<0.001$ \\
\hline MR severe vs mild & 8.43 & $2.32-30.80$ & 0.001 \\
\hline MR moderate vs. mild & 1.50 & $0.60-3.50$ & 0.35 \\
\hline
\end{tabular}

$A F-$ atrial fibrillation, $M R$ - mitral regurgitation.

In multivariate analysis the independent predictors of AF event were: history of previous episode of AF and occurrence of severe (III) in comparison to mild (I) mitral regurgitation (Table II).

The cumulative probability of survival without the onset of AF was significantly different for various modes of pacing: ICD-VR devices -0.71 ; CRT-D - 0.63; and ICD-DR - 0.40 (Figure 1).

The log-rank test result ( $p=0.018)$ indicates that the curves are not homogeneous; in particular the curve for the ICD-DR group is statistically significantly different from the curve for the ICDVR group (ICD-DR vs. ICD-VR: $p=0.02$ ) (CRT-D vs. ICD-DR: $p=0.53$, CRT-D vs. ICD-VR: $p=0.30$ ).

All patients were treated in accordance with the guidelines. It was not demonstrated that the drugs used by the patients significantly influenced the risk of $A F$.

In the group of patients with history of AF who also had AF episodes during follow-up, 13 patients were treated with anticoagulants before enrolment. During the follow-up period, 14 patients who experienced de-novo AF episodes were prescribed with anticoagulant. The remaining patients - 27 (50\%) people - despite episodes of $A F$ in $E G M$ did not receive anticoagulation therapy. They were patients with single and short (less than $5 \mathrm{~min}$ ) episodes of asymptomatic AF. No stroke or transient ischemic episode was observed in any patient during follow-up.

The cumulative probability of occurrence of AF during follow-up in the group of patients with a history of AF episodes is more than twice as high as in the group of patients without these incidents in the

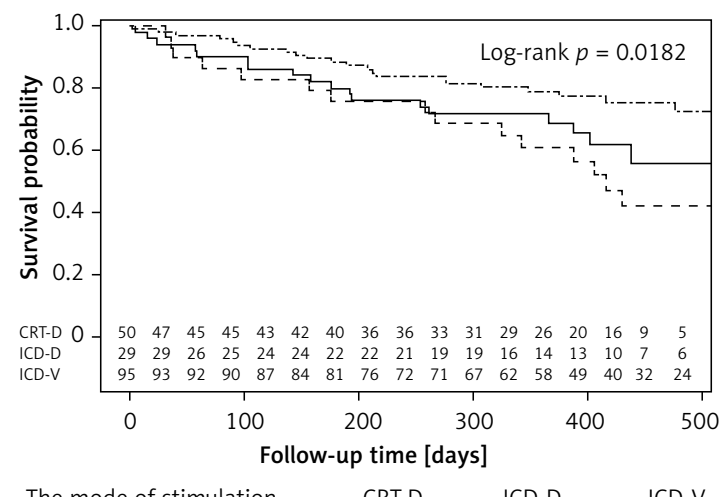

The mode of stimulation _CRT-D - - ICD-D _-.-ICD-V

Figure 1. Probability of survival without an atrial fibrillation attack, estimated by the Kaplan-Meier method depending on the mode of pacing. The curves are not homogeneous $(p=0.018)$ past (log-rank: $p=0.0002$ ). The probability of survival without $A F$ for the entire observation period (on 430 days) in these groups is 0.36 and 0.78 (Figure 2).

During the observation period, 9 (5\%) people died. Table III contains the results of the comparison of the demographic and clinical characteristics of patients who died and who survived during the observation period.

One-dimensional and multidimensional analysis showed that the risk of death in patients with chronic kidney disease is nearly seven times greater than the risk of death in those in whom the disease does not occur (Table IV).

\section{Discussion}

Improved diagnosis of and treatment for AF can have a significant impact on stroke burden [3]. Asymptomatic $A F$ is currently of interest to both cardiology and neurology as a possible cause of the so-called embolic strokes of unknown source [4]. Recently, there have been many publications concerning the detection of atrial high rate episodes (AHRE) in devices and their clinical significance $[5,6]$; in this study, among others, these episodes were taken into account.

AF detection in dual-chamber devices is much simpler, but in single chamber devices it is also not impossible, especially if the person checking the device has extensive experience in this field.

However, it is burdened with higher risk of an error. The value of ventricular high-rate episodes (VHR) in the pacemaker is already a validated tool for measuring AF burden identifying a high-risk

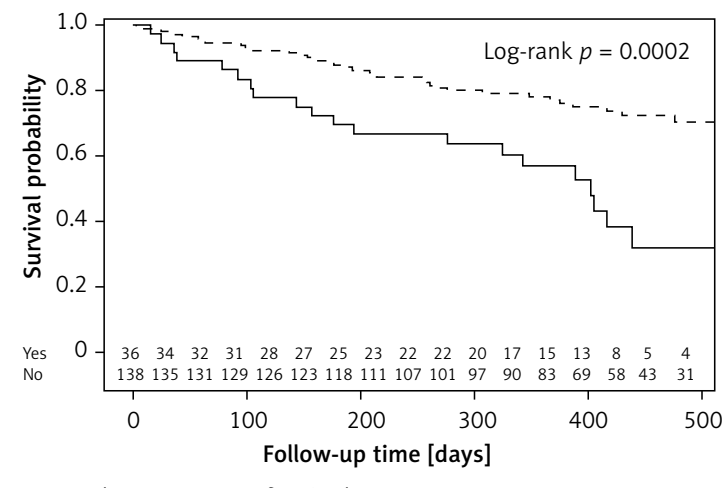

The occurrence of AF in the past - Yes - - No

Figure 2. Event-free survival according to the history of AF in the past. Survival curves represent the time from the beginning of the study up to 475 days 
Table III. Demographic and clinical characteristics of patients depending on their survival

\begin{tabular}{|lccc|}
\hline Parameter & $\begin{array}{c}\text { Survival } \\
n=165(95 \%)\end{array}$ & $\begin{array}{c}\text { Death } \\
n=9(5.0 \%)\end{array}$ & $P$-value \\
\hline Age & $64.2 \pm 10.1$ & $65.8 \pm 7.5$ & 0.65 \\
\hline Male gender & $121(73.3 \%)$ & $6(66.7 \%)$ & 0.70 \\
\hline Chronic ischemic heart disease & $118(71.5 \%)$ & $5(55.6 \%)$ & 0.45 \\
\hline Hypertension & $97(58.8 \%)$ & $7(77.8 \%)$ & 0.32 \\
\hline Diabetes & $60(36.4 \%)$ & $4(44.4 \%)$ & 0.73 \\
\hline Chronic kidney disease & $56(33.9 \%)$ & $7(77.8 \%)$ & 0.01 \\
\hline COPD & $21(12.7 \%)$ & $0(0.0 \%)$ & 0.60 \\
\hline History of stroke & $18(10.9 \%)$ & $0(0.0 \%)$ & 0.60 \\
\hline Ischemic cardiomyopathy & $113(68.5 \%)$ & $5(55.6 \%)$ & 0.47 \\
\hline Ejection fraction & $34.0 \pm 10.8$ & $26.1 \pm 6.5$ & 0.03 \\
\hline LVEDD & $6.0 \pm 1.0$ & $6.7 \pm 1.2$ & 0.049 \\
\hline Left atrium size & $4.6 \pm 0.7$ & $4.7 \pm 0.8$ & 0.51 \\
\hline Severe mitral regurgitation & $65(39.4 \%)$ & $7(77.8 \%)$ & 0.03 \\
\hline NYHA III/IV class & $70(42.4 \%)$ & $7(77.8 \%)$ & 0.08 \\
\hline Prior history of AF & $32(19.4 \%)$ & $4(44.4 \%)$ & 0.09 \\
\hline CRT-D & $47(28.5 \%)$ & $3(33.3 \%)$ & 1.00 \\
\hline ICD-DR & $28(17.0 \%)$ & $1(11.1 \%)$ & \\
\hline ICD-VR & $90(54.5 \%)$ & $5(55.6 \%)$ & \\
\hline CDPD & & & \\
\hline
\end{tabular}

COPD - chronic obstructive pulmonary disease, LVEDD - left ventricular end-diastolic dimension.

Table IV. Results of one-dimensional and multidimensional logistic regression

\begin{tabular}{|c|c|c|c|c|}
\hline \multirow[t]{2}{*}{ Parameter } & \multicolumn{2}{|c|}{ One-dimensional analysis } & \multicolumn{2}{|c|}{ Multidimensional analysis } \\
\hline & Odds ratio $(95 \% \mathrm{Cl})$ & $P$-value & Odds ratio $(95 \% \mathrm{Cl})$ & $P$-value \\
\hline Previous AF & $3.32(0.85-13.09)$ & 0.086 & - & \\
\hline Chronic renal failure & $6.81(1.37-33.88)$ & 0.019 & $6.81(1.37-33.88)$ & 0.019 \\
\hline LVEF & $0.91(0.83-0.99)$ & 0.029 & - & \\
\hline LVEDD & $1.81(0.99-3.31)$ & 0.054 & - & \\
\hline MR severe vs small & $5.38(1.09-26.73)$ & 0.039 & - & \\
\hline NYHA III/IV vs. I/II & $4.75(0.96-23.56)$ & 0.057 & - & \\
\hline
\end{tabular}

$A F$ - atrial fibrillation, LVEF - left ventricular ejection fraction, LVEDD - left ventricular end-diastolic dimension, MR - mitral regurgitation.

subgroup of patients with cardiac implantable electronic devices (CIEDs) [7, 8]; that is why in study this diagnostic algorithm also was used to detect AF episodes.

It is underlined that a prior history of paroxysmal atrial fibrillation, regardless of the time of observation, was associated with a statistically increased risk of AF during follow-up - "AF begets $A F "[9,10]$.

In standard ECG nearly half the patients (46\%) with an implanted dual chamber pacemaker were diagnosed with AF but analysis of the IEGM records showed episodes in the majority of patients (88\%) [11]. For comparison, in the present study, AF occurrence, confirmed by IEGM records, was observed in more than half (51.7\%) of patients with ICD-DR.

The CRYSTAL AF study (Cryptogenic Stroke and Underlying AF) $[12,13]$ showed that with increas- ing follow-up time, the number of AF episodes increases steadily and $74 \%$ of episodes were clinically silent. Although they were no patients with heart failure and cardioverter-defibrillators, the information indicated the scale of the problem of silent AF in selected groups of patients.

In the group of observed patients, the independent risk factors increasing the probability of $\mathrm{AF}$ were only AF in the medical history and severe mitral regurgitation. The obtained results cannot be unequivocally compared with risk factors, which were defined in the literature as predisposing to the occurrence of $A F$, because they were evaluated for the general population including patients with heart failure and preserved ejection fraction. We still do not know if the same risk factors identified in the general population can be applied to patients with heart failure [14]. 
Probably the use of parameters such as the patient's body mass index (BMI), BNP/NT-pro-BNP or MFAP4 and atrial MFAP4 protein expression levels could help to more accurately determine patients predisposed to AF episodes [15-17].

Statistical significance was also obtained for disturbances of the atrioventricular and intraventricular conduction. However, referring to the results of other publications $[18,19]$ sinus node dysfunction as well as conduction disturbances among patients with paroxysmal AF are more frequent than in the general population.

Despite the predominant use of statins in atherothrombotic stroke patients, one study demonstrated better prognosis for patients with AF who were treated with statins and suffered an ischemic stroke [20]. In the current study, over $80 \%$ of patients were treated with statins. There was no statistically significant difference in statin treatment in the group of patients who experienced AF compared to the group of patients who had an episode of AF.

Risk of death in the observed group was statistically significantly higher in patients with chronic kidney disease, which was also confirmed in other studies in patients with ischemic and non-ischemic cardiomyopathy and implanted CRT-D [21].

In conclusion, despite optimal medical therapy in heart failure patients, the incidence of recurrent $A F$ is high and the majority of episodes are asymptomatic. Analysis of device memory contributes to AF diagnosis. Single chamber ICD recipients need other diagnostic tools, new algorithms or devices for reliable AF detection. Remote monitoring should be especially recommended for the subgroup of patients with AF risk factors (conduction disturbances and moderate-to severe mitral insufficiency) for faster detection of this arrhythmia.

In the prospective outpatient study presented, almost half of the patients had single-chamber ICDs implanted, without the possibility of direct registration of atrial ECGs. The diagnosis of atrial arrhythmias in this group of patients is related to the way of programming ventricular arrhythmia detection (the lowest detection zone) and the efficiency of the differentiating algorithms in a given device, as well as the physician conducting follow-up visits having many years of experience.

Data on AF symptoms were collected retrospectively during follow-up visits. With the 30-second AF diagnosis criterion accepted in the paper, some patients (especially among the elderly) may not remember the onset of symptoms. Despite this, between visits the patients had to record all events regarding possible arrhythmia symptoms.

\section{Conflict of interest}

The authors declare no conflict of interest.

\section{References}

1. Otterstad JE, Froeland G, St John Sutton M, Holme I. Accuracy and reproducibility of biplane two-dimensional echocardiographic measurements of left ventricular dimensions and function. Eur Heart J 1997; 18: 507-13.

2. Lancellotti P, Moura L, Pierard LA, et al. European Association of Echocardiography. European Association of Echocardiography recommendations for the assessment of valvular regurgitation. Part 2: mitral and tricuspid regurgitation (native valve disease). Eur J Echocardiogr 2010; 11: 307-32.

3. GBD 2016 Stroke Collaborators. Global, regional, and national burden of stroke, 1990-2016: a systematic analysis for the Global Burden of Disease Study 2016. Lancet Neurol 2019; 18: 439-58.

4. Bernstein RA, Kamel H, Granger CB, Kowal RC, Ziegler PD, Schwamm LH. Stroke of known cause and underlying atrial fibrillation (STROKE-AF) randomized trial: design and rationale. Am Heart J 2017; 190: 19-24.

5. Mahajan R, Perera T, Elliott AD, et al. Subclinical device-detected atrial fibrillation and stroke risk: a systematic review and meta-analysis. Eur Heart J 2018; 39: 1407-15.

6. Van Gelder IC, Healey JS, Crijns HJGM, et al. Duration of device-detected subclinical atrial fibrillation and occurrence of stroke in ASSERT. Eur Heart J 2017; 38: 1339-44.

7. Boriani G, Diemberger I, Ziacchi M, et al. AF burden is important - fact or fiction? Int J Clin Pract 2014; 68: 444-52.

8. Boriani G, Pettorelli D. Atrial fibrillation burden and atrial fibrillation type: clinical significance and impact on the risk of stroke and decision making for long-term anticoagulation. Vascul Pharmacol 2016; 83: 26-35.

9. Rostock T, Steven D, Lutomsky B, et al. Atrial fibrillation begets atrial fibrillation in the pulmonary veins on the impact of atrial fibrillation on the electrophysiological properties of the pulmonary veins in humans. J Am Coll Cardiol 2008; 51: 2153-60.

10. Lu Z, Scherlag BJ, Lin J, et al. Atrial fibrillation begets atrial fibrillation: autonomic mechanism for atrial electrical remodelling induced by short-term rapid atrial pacing. Circ Arrhythm Electrophysiol 2008; 1: 184-92.

11. Israel CW, Grönefeld G, Ehrlich JR, Li YG, Hohnloser SH. Long-term risk of recurrent atrial fibrillation as documented by an implantable monitoring device: implications for optimal patient care. J Am Coll Cardiol 2004; 43: 47-52.

12. Sanna T, Diener HC, Passman RS, et al. Cryptogenic stroke and underlying atrial fibrillation. N Engl J Med 2014; 370: 2478-86.

13. Choe WC, Passman RS, Brachmann J, et al. A comparison of atrial fibrillation monitoring strategies after cryptogenic stroke. Am J Cardiol 2015; 116: 889-93.

14. Targoński R, Sadowski J, Romaszko J, Cichowski L. Identification of clinical risk factors of atrial fibrillation in congestive heart failure. Cardiol J 2013; 20: 364-9.

15. Lip GYH, Merino JL, Banach M, et al. Impact of body mass index on outcomes in the edoxaban versus war- 
farin therapy groups in patients underwent cardioversion of atrial fibrillation (from ENSURE-AF). Am J Cardiol 2019; 123: 592-7.

16. Michalska-Kasiczak M, Bielecka-Dabrowa A, von Haehling S, Anker SD, Rysz J, Banach M. Biomarkers, myocardial fibrosis and co-morbidities in heart failure with preserved ejection fraction: an overview. Arch Med Sci 2018; 14: 890-909.

17. Zhang X, Li H, Kou W, et al. Increased plasma microfibrillar-associated protein 4 is associated with atrial fibrillation and more advanced left atrial remodeling. Arch Med Sci 2019; 15: 632-40.

18. Gomes JA, Kang PS, Matheson M, Gough WB Jr, El-Sherif $\mathrm{N}$. Coexistence of sick sinus rhythm and atrial flutter-fibrillation. Circulation 1981; 63: 80-6.

19. van den Berg MP, van Gelder IC. Atrial fibrillation and sinus node dysfunction. J Am Coll Cardiol 2001; 38 1585-6.

20. Kotlęga D, Gołąb-Janowska M, Meller A, et al. Beneficial effects of pre-stroke statins use in cardioembolic stroke patients with atrial fibrillation: a hospital-based retrospective analysis. Arch Med Sci 2019; 15: 385-92.

21. Beiert T, Straesser S, Malotki R, Stöckigt F, Schricke JW, Andrié RP. Increased mortality and ICD therapies in ischemic versus non-ischemic dilated cardiomyopathy patients with cardiac resynchronization having survived until first device replacement. Arch Med Sci 2019; 15: $845-56$. 